\title{
A Wideband, Low-Noise Superconducting Amplifier with High Dynamic Range
}

\author{
Byeong Ho Eom ${ }^{\dagger}$, Peter K. Day*, Henry G. LeDuc*, Jonas Zmuidzinas ${ }^{\dagger *}$,
}

(Dated: January 12, 2012)

${ }^{\dagger}$ California Institute of Technology, Pasadena CA 91125, USA

* Jet Propulsion Laboratory, California Institute of Technology, Pasadena CA 91109, USA

Amplifiers are ubiquitous in electronics and play a fundamental role in a wide range of scientific measurements. From a user's perspective, an ideal amplifier has very low noise, operates over a broad frequency range, and has a high dynamic range - it is capable of handling strong signals with little distortion. Unfortunately, it is difficult to obtain all of these characteristics simultaneously. For example, modern transistor amplifiers offer multi-octave bandwidths and excellent dynamic range. However, their noise remains far above the fundamental limit set by the uncertainty principle of quantum mechanics.[1] Parametric amplifiers, which predate transistor amplifiers and are widely used in optics, exploit a nonlinear response to transfer power from a strong pump tone to a weak signal. If the nonlinearity is purely reactive, i.e. nondissipative, in theory the amplifier noise can reach the quantum-mechanical limit.[2] Indeed, microwave frequency superconducting Josephson parametric amplifiers $[3,4]$ do approach the quantum limit, but generally are narrow band and have very limited dynamic range. In this paper, we describe a superconducting parametric amplifier that overcomes these limitations. The amplifier is very simple, consisting only of a patterned metal film on a dielectric substrate, and relies on the nonlinear kinetic inductance of a superconducting transmission line. We measure gain extending over $2 \mathrm{GHz}$ on either side of an $11.56 \mathrm{GHz}$ pump tone, and we place an upper limit 


\section{on the added noise of the amplifier of 3.4 photons at $9.4 \mathrm{GHz}$. Furthermore,}

the dynamic range is very large, comparable to microwave transistor amplifiers, and the concept can be applied throughout the microwave, millimeter-wave and submillimeter-wave bands.

Over the past decade, the combination of high-performance superconducting microresonators and low-noise, microwave frequency cryogenic transistor amplifier readouts has proven to be particularly powerful for a wide range of applications including photon detection and quantum information experiments. [5-7] These developments have generated strong renewed interest in superconducting amplifiers that achieve even lower readout noise. [8-12]. Most of these devices are parametric amplifiers that make use of the nonlinear inductance of the Josephson junction, which is almost ideally reactive with little dissipation below the critical current $I_{c}$. As a result, Josephson paramps can be exquisitely sensitive, approaching the standard quantum limit of half a photon $\hbar \omega / 2$ of added noise power per unit bandwidth in the standard case when both quadratures of a signal at frequency $\omega$ are amplified equally. Here $\hbar$ is Planck's constant divided by $2 \pi$. Even less noise is possible in situations when only one quadrature is amplified.[1]. In comparison, the added noise of cryogenic transistor amplifiers is typically 10-20 times the quantum limit.[13] However, the dynamic range of Josephson paramps is regulated by the Josephson energy $E_{J}=\hbar I_{c} / 4 \pi e$ (here $e$ is the electron charge) to values that are far lower than for transistor amplifiers. Furthermore, as in optical parametric oscillators in which light passes many times through a nonlinear medium, previous superconducting paramps generally use resonant circuits to enhance the effective nonlinearity in order to achieve high gain. Consequently, amplification is achieved over a narrow instantaneous frequency range, typically of order a few $\mathrm{MHz}$, vs. $\sim 10 \mathrm{GHz}$ for transistor amplifiers. This results in a relatively slow response time that can hinder observa-

tion of time-dependent phenomena, e.g. quantum jumps.[14] Also, as with superconducting quantum interference devices (SQUIDs), the combination of a limited dynamic range and a limited bandwidth results in a low Shannon information capacity and limits the utility of 
Josephson paramps for multiplexed readout of detector arrays.[15]

Instead of using a resonator, the optical or electrical path may be unfolded into a long nonlinear transmission line or waveguide. This results in a traveling wave paramp[16, 17] which has a very broad intrinsic bandwidth. However, a low-dissipation medium that is sufficiently nonlinear over a realizable length must be found. At visible and infrared wavelengths, these requirements are met in optical fibers[18] and silicon waveguides[19] through the nonlinear process of four-wave mixing (FWM) that results from the intensity dependence of the refractive index, i.e. the Kerr effect. Fiber paramps achieve high (>60 dB) gain[18] and single-quadrature versions have exhibited noise levels below the standard quantum limit.[20] An analogous microwave device using a metamaterial of numerous Josephson junctions embedded in a transmission line[21, 22] has been proposed and investigated, but this design has not yet resulted in a practical amplifier.

Like a Josephson junction, a thin superconducting wire behaves as a nondissipative inductor for currents below a critical current $I_{c}$. The critical current is therefore an obvious scale for nonlinear behavior in both junctions and wires, although $I_{c}$ for a wire is typically orders of magnitude larger than for a junction. Indeed, the phenomenological GinzburgLandau theory and the microscopic BCS theory $[23,24]$ both predict the nonlinearity of the kinetic inductance of superconductors. In practice this nonlinearity is usually weak, though resonant paramps based on this effect have been proposed and investigated[25, 26]. Here we show that the use of a high-resistivity superconductor such as TiN[27] or NbTiN results in a kinetic inductance nonlinearity that is sufficient to allow parametric gain in a practical, realizable traveling wave geometry.

The current variation of the kinetic inductance of a superconducting wire is expected to be quadratic to lowest order, i.e. $L_{k}(I) \approx L_{k}(0)\left[1+\left(I / I_{*}\right)^{2}\right]$, just as in the case of Josephson junctions. The Mattis Bardeen theory gives $L_{k}(0)=\hbar R_{n} / \pi \Delta$ for a wire with normal state resistance $R_{n}$ and superconducting gap parameter $\Delta$ and whose transverse dimensions are small enough so that the current distribution is approximately uniform. $I_{*}$ is comparable to 
$I_{c}$, and can be roughly estimated by equating the kinetic energy of the Cooper pairs $L_{k} I^{2} / 2$ to the pairing energy $E_{p}=2 N_{0} \Delta^{2} V$, where $N_{0}$ is the density of states at the Fermi level and $V$ is the volume. The resulting expression for $I_{*}^{2}$ is proportional to $1 / R_{n}$. The phase velocity of the transmission line is $v_{p h}=1 / \sqrt{\mathcal{L C}} \approx v_{p h}(0)\left(1-\alpha I^{2} / 2 I_{*}^{2}\right)$, where $\mathcal{L}$ and $\mathcal{C}$ are the total inductance and capacitance per unit length, and $\alpha$ is the ratio of kinetic inductance to total inductance. The magnitude of the Kerr effect in the line is therefore proportional to $\alpha / I_{*}^{2}$ and is enhanced in films with high normal state resistivity $\rho_{n}$ due both to large $\alpha$ and small $I_{*}$. The TiN and NbTiN films produced in our laboratory have $\rho_{n} \approx 100 \mu \Omega \mathrm{cm}$, nearly three orders of magnitude larger than for typical aluminum films, and have very low microwave loss in the superconducting state.[27] The high resistivity also results in a large penetration depth,[7] in the range $2-20 \mu \mathrm{m}$ depending on thickness (20-50 $\mathrm{nm}$ typically) and critical temperature $T_{c}$, so a uniform current density is readily achieved in our micron-scale wires patterned by optical lithography.

The nonlinear kinetic inductance is illustrated in fig. 1, which shows the phase shift of a microwave tone passing through an NbTiN transmission line as a function of the DC current flowing through it. In fact, such a current-controlled phase shifter has been previously proposed[28] but not successfully demonstrated due to an increase in the microwave dissipation with DC current. Similar dissipative behavior has also been noted in superconducting thin film resonators (see supplementary information). In contrast, no increase in dissipation was observed for the range of phase shifts displayed in fig. 1. Also, provided that the temperature is kept well below the critical temperature $T_{c}$, our TiN[27] and NbTiN microresonators remain nondissipative for microwave currents sufficiently strong to develop a significant reactive nonlinearity. These results are described in the supplementary information.

The parametric gain produced through FWM can be calculated using coupled mode equations that have been developed to describe optical fiber paramps (see supplementary information). In general, FWM may involve two separate pump tones, but we consider only the degenerate case where the pump frequencies are equal. The equations then describe the 
interaction of the pump at angular frequency $\omega_{p}=2 \pi f_{p}$, signal at $\omega_{s}$, and the generated idler tone at $\omega_{i}=2 \omega_{p}-\omega_{s}$. For a dispersionless line, which is a good approximation for a uniform superconducting transmission line at frequencies well below the gap frequency $2 \Delta / h$, the signal and idler gains for $f_{s} \approx f_{p}$ are $G_{s}=1+(\Delta \theta)^{2}$ and $G_{i}=(\Delta \theta)^{2}$, where $\Delta \theta$ is the nonlinear phase shift, in radians, of the pump tone due to its own $\mathrm{AC}$ current. Fig. 1 shows that phase shifts of several radians can be achieved in response to a DC current. Comparable phase shifts can also be achieved in response to RF currents, as described in the supplementary information. Dispersion, due either to material or waveguide properties, controls the phase slippage between the waves as they propagate, which in turn determines whether the signal is amplified or deamplified. The linear dispersion $\Delta \beta=\beta\left(\omega_{S}\right)+\beta\left(\omega_{I}\right)-$ $2 \beta\left(\omega_{P}\right)$ involves a difference of the propagation constants $\beta(\omega)$ for small-amplitude waves at the signal, idler and pump frequencies, and vanishes for a dispersionless line obeying $\beta(\omega)=$ $\omega / \bar{c}$. The coupled-mode equations predict that maximum gain occurs when $\Delta \beta=-2 \Delta \theta / L$ because this value of linear dispersion compensates for the phase slippage that arises from the nonlinearity. Furthermore, the resulting gain $G_{s}=\exp (2 \Delta \theta) / 4$ varies exponentially with line length $L$ rather than quadratically[18, 29]. For much larger or smaller values of $\Delta \beta$, loss of phase match yields low gain.

In fact, some amount of dispersion is necessary, because a dispersionless Kerr medium leads to generation of a shock front when $\Delta \theta \gtrsim 1$, preventing significant parametric gain.[30] Indeed, a superconducting line generates the third harmonic $3 f_{p}$ due to the voltage term $I^{2} d I / d t$ arising from the nonlinear inductance. This is the first step in the formation of the shock front: once the $3 f_{p}$ harmonic is present, other harmonics can be generated. This problem is dealt with in a simple manner using dispersion engineering, leading to the device shown in fig. 1. Periodic perturbations are included in the coplanar waveguide (CPW) transmission line with a separation corresponding to half of a wavelength at frequency $f_{\text {per }}$, with $f_{\text {per }}$ slightly larger than $3 f_{p}$. Much like an electronic or photonic bandgap, this results in a stop band centered at $f_{\text {per }}$ that includes $3 f_{p}$, blocking harmonic generation. We also 
slightly alter every third perturbation, resulting in weak stop bands around $f_{\text {per }} / 3$ and $2 f_{\text {per }} / 3$ (fig. 1c). This gives rise to localized dispersion features near these frequencies; fine-tuning of the pump frequency $f_{p}$ in the vicinity of these features allows the optimum value of $\Delta \beta$ to be achieved for a wide range of signal frequencies. For the device shown in fig. 1, the stop bands occur at at multiples of $f_{\text {per }} / 3 \approx 5.9 \mathrm{GHz}$, not far from the design value of $5 \mathrm{GHz}$. We chose to operate the the device with the pump tuned near $2 f_{\text {per }} / 3 \approx 11.8 \mathrm{GHz}$ because this dispersion feature was stronger; note that a strong stop band also occurs at $3 \times 11.8 \mathrm{GHz}$, which prevents formation of pump harmonics.

We measured the gain of the amplifier with the pump tuned to $11.56 \mathrm{GHz}$ using the circuit shown in fig. 2, excluding the bandpass filter. The gain increases with pump power until a critical pump power is reached, which we identify with the onset of nonlinear dissipation in the line. Significant gain is observed over a frequency range of approximately $8 \mathrm{GHz}$ to $14 \mathrm{GHz}$, with a notch around the pump frequency (fig. 3). A theoretical gain curve (fig. 3, top), generated by integrating the coupled mode equations and including a model of the loaded line dispersion (see supplementary info), is in rough agreement with the average gain. However, there is a fine-scale variation of the gain that is approximately periodic with signal frequency, and this gain ripple increases with pump power and has a divergent behavior around the critical pump power. The average gain at the higher pump power shown is $10 \mathrm{~dB}$, with several peaks extending above $20 \mathrm{~dB}$. From the frequency spacing, it is clear that the gain ripple is due to standing waves created by reflections at the ends of the line. The reflections are probably caused by the non-optimal on-chip tapered impedance transformers, or possibly the wirebond transitions or other components near the device.

For the purpose of measuring the noise of the paramp, we chose a signal frequency of $f_{s}=9.37 \mathrm{GHz}$, close to a gain peak . The output power of the amplifier chain was measured in a narrow band around $f_{s}$ using a spectrum analyzer. A cryogenic switch (fig. 2) was used to calibrate the system. The added noise power per unit bandwidth of the high electron mobility transistor $(\mathrm{HEMT})$ at $4.1 \mathrm{~K}$ was measured to be $A_{\mathrm{HEMT}}=16.1$, in photon units 
$(\hbar \omega)$, by switching between between $50 \Omega$ resistors on the $4.1 \mathrm{~K}$ and base temperature stages of the dilution refrigerator (switch positions 2 and 3). With the paramp connected to the HEMT (position 1), we make measurements with the pump tone on and off (fig. 4). Because the gain peak shifts to lower frequency when the pump is applied, we slightly reduce the signal frequency to remain at the peak. Since the transmission of the measurement system should remain constant over this small frequency interval, the increase in the output signal power gives the gain of the paramp, $G_{\mathrm{PA}}=18.6 \mathrm{~dB}$. It is evident that the paramp is considerably less noisy than the HEMT, because the signal-to-noise ratio improves by $7.8 \mathrm{~dB}$ with the pump turned on (fig. 4). The paramp noise may be quantitatively determined from the ratio $R=\left(n_{\text {on }}-n_{0}\right) /\left(n_{\text {off }}-n_{0}\right)$, where $n_{\text {on }}$ and $n_{\text {off }}$ are the measured noise levels with pump on and off, and $n_{0}$ is the noise from sources after the HEMT and is found by turning off the HEMT. However, with the paramp connected to the HEMT (switch position 1) and no pump applied, we find that the noise floor is slightly higher than with the HEMT connected to the cold load. This increase in the system added noise is $A_{\text {sys }}=2.9$. We have not yet determined the origin of this noise and therefore we do not know the gain $G_{\text {sys }}$ this noise experiences when the pump is turned on. As a result, when we calculate the noise added by the paramp,

$$
A_{\mathrm{PA}}=\frac{R-1}{G_{\mathrm{PA}}^{\prime}} A_{\mathrm{H}}+\frac{R-G_{\mathrm{sys}}}{G_{\mathrm{PA}}^{\prime}} A_{\mathrm{sys}}+\frac{R}{2 G_{\mathrm{PA}}^{\prime}}-\frac{1}{2}
$$

we obtain a range of values $1.1 \leq A_{\mathrm{PA}} \leq 3.4$, corresponding to $1 \leq G_{\mathrm{sys}} \leq G_{\mathrm{PA}}^{\prime}$. Here $G_{\mathrm{PA}}^{\prime}$ accounts for the isolator loss (fig. 2).

To confirm the noise measurement, we used a variable-temperature $50 \Omega$ resistor connected to the paramp input through a $3 \mathrm{~dB}$ hybrid coupler (fig. 4). The slope of the linear relationship between output noise and resistor temperature calibrates the gain of the system, and extrapolating this relationship to zero resistor temperature gives the added noise of the amplifier plus any additional input noise. If we assume that only vacuum noise $\hbar \omega / 2$ is present at the input, the added noise of the paramp is $3.3 \pm 0.2$ photons, in agreement with the previous measurement. 
The dynamic range of the paramp was investigated when operating with $18 \mathrm{~dB}$ signal gain under the same conditions as used for the noise measurement. The system gain compressed by $1 \mathrm{~dB}$ for a signal power at the paramp input of around $100 \mathrm{pW}$. However, measurements made with the pump turned off show that the HEMT, rather than the paramp, is reponsible for this gain compression. Theoretically, saturation of the paramp occurs when the amplified signal power becomes a significant fraction of the pump power, around $100 \mu \mathrm{W}$ for this device, so that the pump becomes depleted.

In summary, we have demonstrated a simple and robust superconducting amplifier with very low noise, wide bandwidth, and high dynamic range. In fact, given the extremely low dissipation of the superconductors used,[27] it seems likely that the amplifier is operating very near the quantum limit and that the added noise is due to imperfections of the present measurement system. For example, the image frequency $f_{I}=13.75 \mathrm{GHz}$ is outside the bandwidth of our isolator, so noise from the HEMT may leak back toward the paramp at that frequency and contribute to noise at $f_{s}$. Straightforward design improvements should allow high $(>20 \mathrm{~dB})$ gain to be achieved over an octave of instantaneous bandwidth. Periodic loading structures can readily be designed for operating frequencies in the microwave, millimeter-wave, and submillimeter-wave bands, potentially approaching the gap frequency of the superconducting film $(2 \Delta / h \approx 1.4 \mathrm{THz}$ for $\mathrm{NbTiN})$. The lower frequency limit is determined only by the length of transmission line that can be fabricated. By applying a DC current bias, the amplifier can also be operated in a three-wave mixing mode, where the pump is at twice the average of the signal and idler frequencies. More generally, we hope that our demonstration will serve as a clear illustration of the remarkable nonlinear properties of highly resistive superconductors and will stimulate development of a much broader set of applications, just as was the case for nonlinear optics.

\section{References}

1. Caves, C. M. Quantum limits on noise in linear amplifiers. Phys. Rev. D 26, 1817-1839 (Oct. 1982). 
2. Louisell, W. H., Yariv, A. \& Siegman, A. E. Quantum fluctuations and noise in parametric processes. I. Phys. Rev. 124, 1646-1654 (Dec. 1961).

3. Zimmer, H. Parametric amplification of microwaves in superconducting Josephson tunnel junctions. Appl. Phys. Lett. 10, 193-195 (1967).

4. Moshovich, R. et al. Observation of zero-point noise squeezing via a Josephson parametric amplifier. English. Phys. Rev. Lett. 65, 1419-1422. ISSN: 0031-9007 (1990).

5. Day, P., LeDuc, H., Mazin, B., Vayonakis, A. \& Zmuidzinas, J. A broadband superconducting detector suitable for use in large arrays. Nature 425, 817-821 (2003).

6. Wallraff, A. et al. Strong coupling of a single photon to a superconducting qubit using circuit quantum electrodynamics. Nature 431, 162-167 (Sept. 2004).

7. Zmuidzinas, J. Superconducting microresonators: Physics and applications (in press). Annu. Rev. Cond. Mat. Phys. 3, 15.1-15.46 (2012).

8. Castellanos-Beltran, M. A., Irwin, K. D., Hilton, G. C., Vale, L. R. \& Lehnert, K. W. Amplification and squeezing of quantum noise with a tunable Josephson metamaterial. Nature Physics 4, 929-931 (Dec. 2008).

9. Yamamoto, T. et al. Flux-driven Josephson parametric amplifier. App. Phys. Lett. 93, 042510 (July 2008).

10. Bergeal, N. et al. Phase-preserving amplification near the quantum limit with a Josephson ring modulator. Nature 465, 64-68 (May 2010).

11. Spietz, L., Irwin, K., Lee, M. \& Aumentado, J. Noise performance of lumped element direct current superconducting quantum interference device amplifiers in the $4-8 \mathrm{GHz}$ range. App. Phys. Lett. 97, 142502 (Oct. 2010).

12. Hover, D. et al. Superconducting low-inductance undulatory galvanometer microwave amplifier. ArXiv e-prints. arXiv:1109.5209 [cond-mat.supr-con] (Sept. 2011).

13. Pospieszalski, M. W. Extremely low-noise amplification with cryogenic FETs and HFETs: 1970-2004. IEEE Microwave Magazine, Vol. 6, p. 62-75 6, 62-75 (2005).

14. Vijay, R., Slichter, D. H. \& Siddiqi, I. Observation of quantum jumps in a superconducting artificial atom. Phys. Rev. Lett. 106, 110502 (Mar. 2011).

15. Irwin, K. D. in AIP Conf. Proc. (ed B. Young, B. Cabrera, \& A. Miller) 1185 (Dec. 2009), 229-236. doi:10.1063/1.3292320.

16. Cullen, A. L. A travelling-wave parametric amplifier. Nature 181, 332 (1958).

17. Tien, P. K. Parametric amplification and frequency mixing in propagating circuits. J. App. Phys. 29, 1347-1357 (Sept. 1958).

18. Hansryd, J, Andrekson, P., Westlund, M, Li, J \& Hedekvist, P. Fiber-based optical parametric amplifiers and their applications. IEEE J. Sel. Top. Quantum Electron. 8, 506-520. ISSN: 1077-260X (2002).

19. Foster, M. A. et al. Broad-band optical parametric gain on a silicon photonic chip. Nature 441, 960-963 (June 2006). 
20. Tong, Z. et al. Towards ultrasensitive optical links enabled by low-noise phase-sensitive amplifiers. Nature Photonics 5, 430-436 (July 2011).

21. Sweeny, M. \& Mahler, R. A travelling-wave parametric amplifier utilizing Josephson junctions. IEEE Trans. Magn. 21, 654 (Mar. 1985).

22. Yurke, B., Roukes, M. L., Movshovich, R. \& Pargellis, A. N. A low-noise series-array Josephson junction parametric amplifier. Appl. Phys. Lett. 69, 3078-3080 (Nov. 1996).

23. Parmenter, R. H. Nonlinear electrodynamics of superconductors with a very small coherence distance. RCA Review 23, 323-352 (1962).

24. Anthore, A., Pothier, H. \& Esteve, D. Density of states in a superconductor carrying a supercurrent. Phys. Rev. Lett. 90, 127001 (12 2003).

25. Landauer, R. W. Superconductive Parametric Amplifier U.S. Patent Number 3,111,628. 1963.

26. Tholen, E. A. et al. Nonlinearities and parametric amplification in superconducting coplanar waveguide resonators. English. Appl. Phys. Lett. 90. ISSN: 0003-6951. doi:\{10. 1063/1.2750520\} (2007).

27. Leduc, H. G. et al. Titanium nitride films for ultrasensitive microresonator detectors. App. Phys. Lett. 97, 102509 (Sept. 2010).

28. Anlage, S. M., Snortland, H. J. \& Beasley, M. R. A current controlled variable delay superconducting transmission-line. English. IEEE Trans. Magn. 25, 1388-1391. ISSN: 0018-9464 (1989).

29. Stolen, R. \& Bjorkholm, J. Parametric amplification and frequency conversion in optical fibers. IEEE J. Quantum Electron. 18, 1062 -1072. ISSN: 0018-9197 (1982).

30. Landauer, R. Shock waves in nonlinear transmission lines and their effect on parametric amplification. IBM J. Res. Dev. 4, 391-401. ISSN: 0018-8646 (1960).

\section{Acknowledgements}

The research was carried out at the Jet Propulsion Laboratory, California Institute of Technology, under a contract with the National Aeronautics and Space Administration and has been supported in part by NASA (Science Mission directorate), the Keck Institute for Space Studies and the JPL Research and Technology Development program.

Correspondence and requests for materials should be addressed to P.D. (e-mail: Peter.K.Day@jpl.nasa.gov). 


\section{Figure Legends}

Figure 1 Phase response to DC current and amplifier design. a, This plot illustrates the nonlinearity of the kinetic inductance. A NbTiN coplanar waveguide (CPW) transmission line (shown in panel b) was measured in transmission using a microwave network analyzer. The total phase length was 670 radians at $4 \mathrm{GHz}$. Using bias tees, a DC current was passed down the center conductor. The resulting microwave phase shift (measured at $4 \mathrm{GHz}$ ) displays a quadratic dependence with current. No comparable effect occurred when adjusting the voltage of the center strip relative to the ground planes. This shows that the kinetic inductance has a nonlinear behavior that is well described by $\delta L_{k i n} \propto I^{2}$. b, A picture of the amplifier (left) which consists of a $0.8 \mathrm{~m}$ length of NbTiN CPW line arranged in a double spiral to reduce resonances due to coupling between adjacent lines. The thickness of the line is $35 \mathrm{~nm}$ and the center conductor and gap widths are $1 \mu \mathrm{m}$. At the input and output of the line, the CPW geometry tapers from center strip and gap widths of $30 \mu \mathrm{m}$ and $5 \mu \mathrm{m}$ to adiabatically transform the characteristic impedance of the line from close to $50 \Omega$ to $300 \Omega$. The line is periodically loaded by widening a short section after every length $D=877 \mu \mathrm{m}$ as shown on the right, producing the stop band and dispersion characteristics. The phase velocity on the line is $0.1 \mathrm{c}$ due to its large kinetic inductance. c, An illustration of the the effect of the periodic loading pattern (shown schematically) on the transmission of an infinite transmission line. The gray regions represent stop bands; waves in these frequency ranges decay evanescently. The graph represents the difference between the propagation constant of the line and linear $(\propto \omega)$ dispersion. As the fractional width of the third stop band is much larger than the first, the pump can be placed at a propagating frequency while $3 \omega_{p}$ is blocked.

Figure 2 Circuit for paramp gain and noise measurements. The pump tone is produced by a low phase noise synthesizer ( $<150 \mathrm{dBc}$ at $10 \mathrm{MHz}$ offset), amplified to a suitable level and filtered using a copper cavity mode bandpass filter. After a splitter, the pump tone is attenuated at $4 \mathrm{~K}$ and filtered using a commercial combline filter with a bandwidth 
of $200 \mathrm{MHz}$ around $11.56 \mathrm{GHz}$. This bandpass filter is used for the noise measurements, and provides greater than $70 \mathrm{~dB}$ of attenuation for noise on the pump line at the signal frequencies of interest. The other output of the splitter is phase and amplitude adjusted and used to null the pump tone that passes through the paramp and would otherwise saturate the $4 \mathrm{~K}$ HEMT amplifier. The signal tone is generated either with another synthesizer or a vector network analyzer. Its level is reduced by warm and cold attenuators and coupled to the paramp input using a $20 \mathrm{~dB}$ directional coupler on the base temperature stage of the dilution refrigerator. A cryogenic isolator is used after the paramp to absorb noise radiated toward the paramp by the HEMT post-amplification stage. The noise of the paramp and HEMT is measured with the help of a cryogenic switch after the isolator that can connect the HEMT amplifier to either the paramp or $50 \Omega$ loads at the base temperature and $4 \mathrm{~K}$ stages. The loss of the isolator was measured to be $0.8 \mathrm{~dB}$. The switch has negligible loss. After further amplification at room temperature, the signal is measured using either a spectrum analyzer or the network analyzer. The diagram does not include coax line losses. All coaxes below $4 \mathrm{~K}$ are superconducting.

Figure 3 Measured and calculated gain. (Top) This plot shows a gain profile calculated using the coupled mode equations for FWM including the dispersion of the device determined using a transmission line model of the periodic loading structure. A parameter quantifying the nonlinearity of the line and the frequency shift of the dispersion curve due to the cross phase modulation from the pump tone were adjusted for a reasonable match to the data. (Second and third) these plots show the measured ratio of pump on over pump off transmission (gray lines) for two pump powers (-8.0 and $-9.4 \mathrm{dBm}$ at the input of the paramp). The blue lines are the measured data smoothed by averaging over $60 \mathrm{MHz}$. The large peak at $11.9 \mathrm{GHz}$ is an artifact arising from the shift to lower frequency of the transmission dip produced by the periodic loading. (bottom) The spacing between the gain ripples, shown on an expanded scale, corresponds to the electrical length of the NbTiN transmission line.

Figure 4 Noise compared with the HEMT amplifier . (Left) Increase in signal to 
noise ratio of a weak microwave tone applied to the input of the paramp. With the pump off (green) the noise floor is limited by the HEMT amplifier. With the pump on (blue) the signal gain is $18.6 \mathrm{~dB}$ and signal noise ratio has increased by $7.8 \mathrm{~dB}$. The red lines are a fit to the spectrum analyzer's response to a monochromatic signal. The pump on data were taken with $f_{s}=9.3672 \mathrm{GHz}$, while the pump off data used $f_{s}=9.3845 \mathrm{GHz}$. (Right) Noise referred to the input of the paramp (blue) and the HEMT amplifier (green) in photon units versus the calibrator temperature. The circuit configuration for this measurement is shown in the inset. The $50 \Omega$ resistor is mounted on a temperature controlled stage connected to the mixing chamber and terminates a $0.85 \mathrm{~mm}$ diameter NbTi coax line. The noise signal is added to the pump at the input of the paramp using a $3 \mathrm{~dB}$ hybrid coupler. The total loss between the resistor and the paramp input was $4.1 \pm 0.5 \mathrm{~dB}$, where we have taken the insertion loss of hybrid to lie between the room temperature measured value of $1 \mathrm{~dB}$ and zero. An isolator is used between the noise calibrator and the hybrid to avoid heating from reflected pump power. 

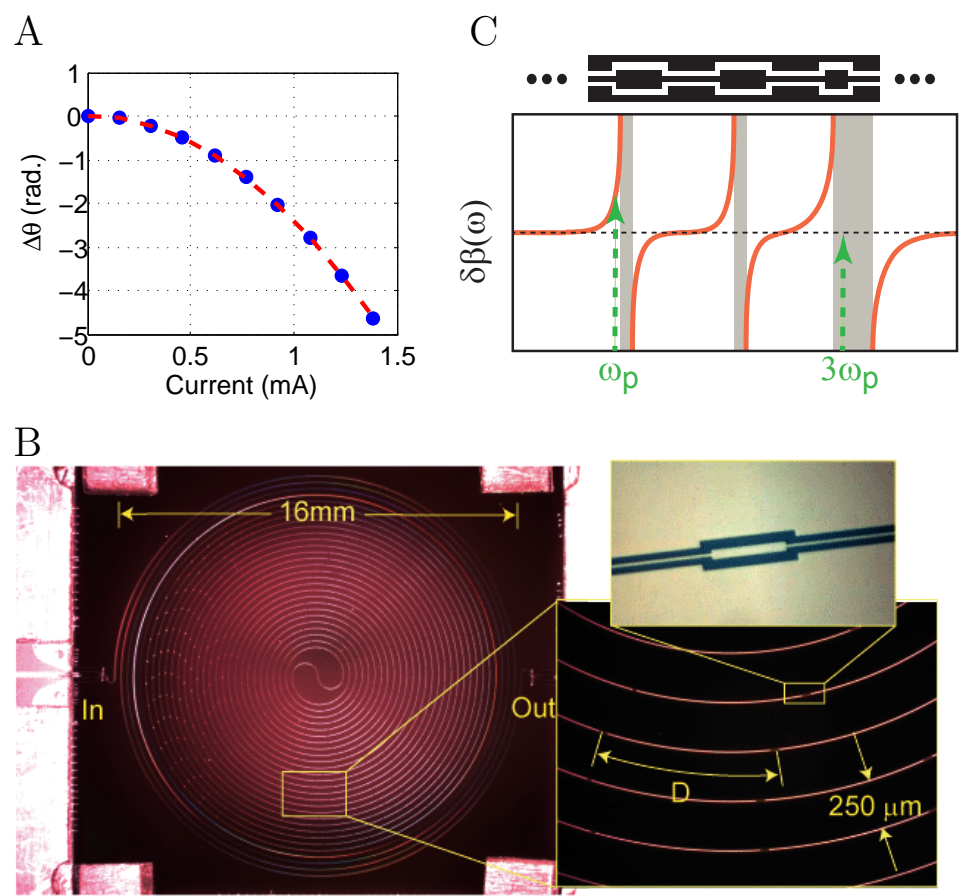

Figure 1: Eom et al.

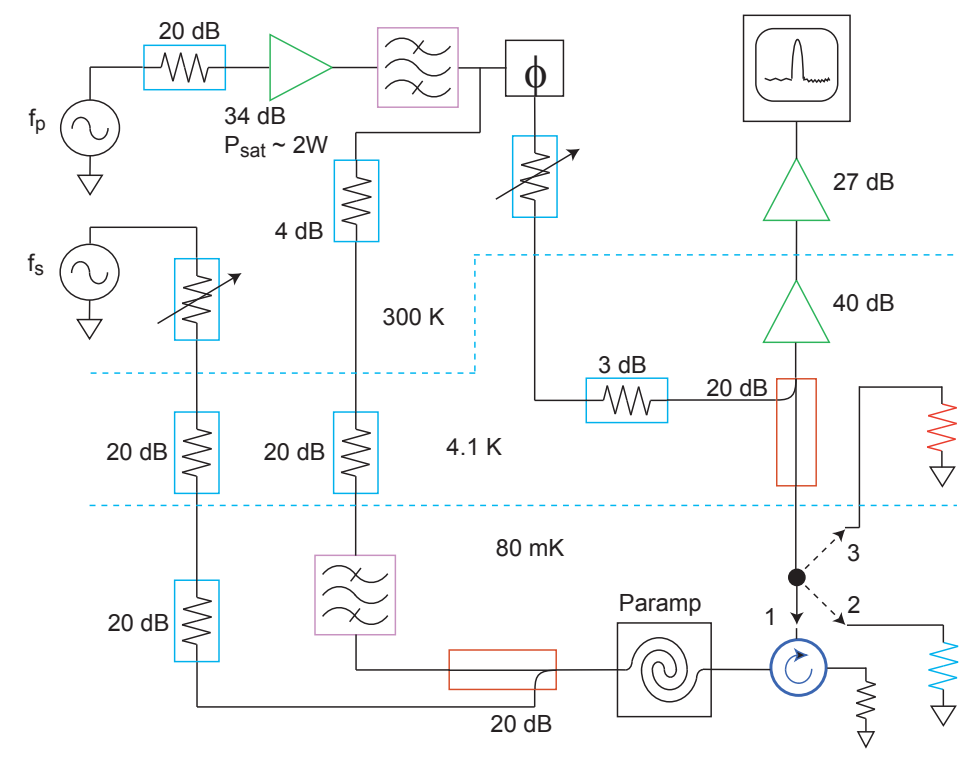

Figure 2: Eom et al. 


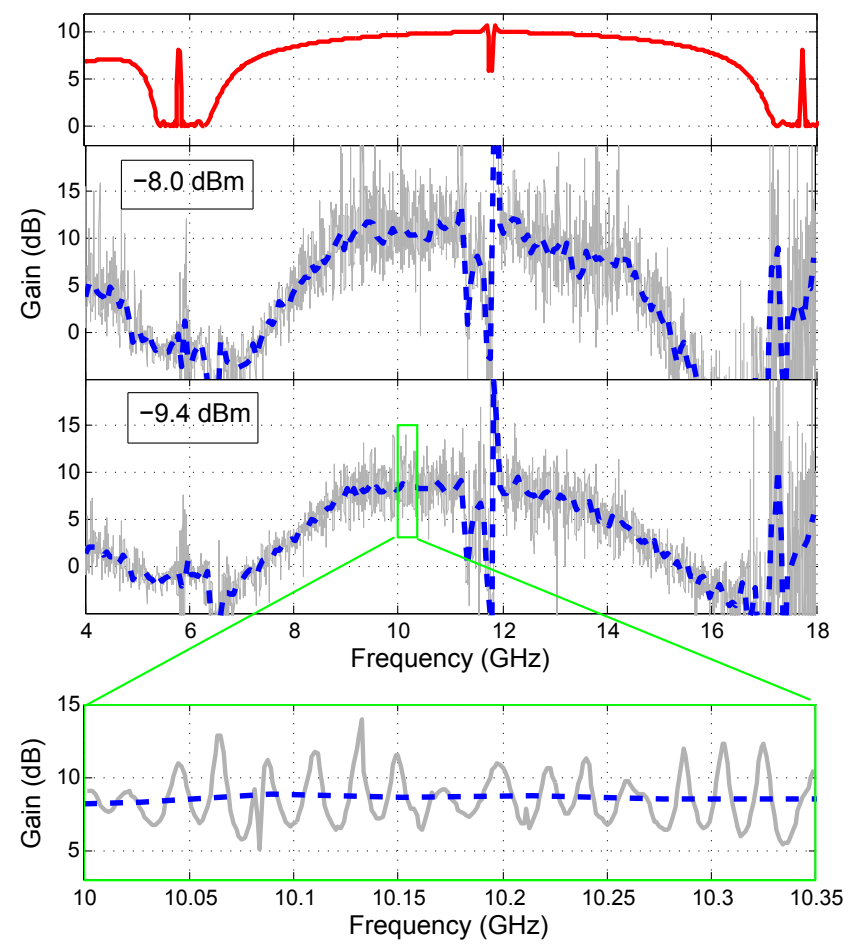

Figure 3: Eom et al.
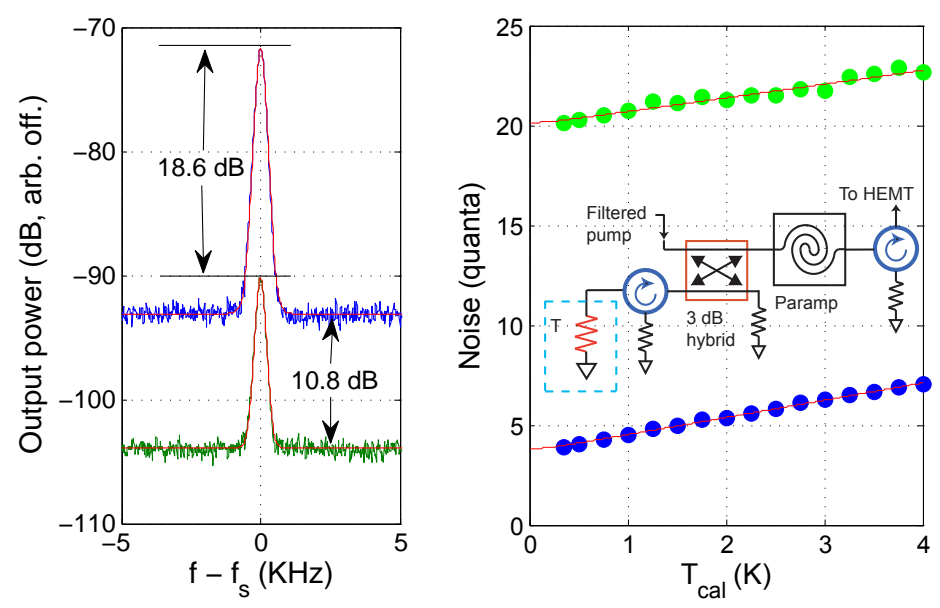

Figure 4: Eom et al. 


\title{
Supplementary Information for: “A Wideband, Low-Noise Superconducting Amplifier with High Dynamic Range"
}

\author{
Byeong Ho Eom ${ }^{\dagger}$, Peter K. Day*, Henry G. LeDuc*, Jonas Zmuidzinas ${ }^{\dagger *}$,
}

(Dated: January 12, 2012)

${ }^{\dagger}$ California Institute of Technology, Pasadena CA 91125, USA

* Jet Propulsion Laboratory, California Institute of Technology, Pasadena CA 91109, USA

\section{Resonator Measurements of the nonlinear response of TiN and NbTiN films}

The dissipation of a system can be sensitively measured using resonant techniques. Here we present measurements of microresonators made from thin TiN and NbTiN films, driven with input powers large enough to cause the resonances to exhibit nonlinear behavior due to the current dependence of the kinetic inductance. Our aim is to examine the relationship between the nonlinear inductance and dissipation in these films.

The measurements were performed on resonators with a "lumped element" geometry described in fig. S1, which were weakly coupled capacitively to a finite width ground plane CPW feedline. The quality factor $Q_{c}$ describing the strength of the feedline coupling was $6 \times 10^{5}$, and the internal quality factors describing resonator losses, $Q_{i}$ were $5 \times 10^{6}$ and $2.5 \times 10^{6}$ for the TiN and NbTiN resonators, respectively. The devices were cooled in a dilution refrigerator to below $100 \mathrm{mK}$.

The resonance curves were measured with a homodyne detection circuit (fig. S1). The driving signal was produced by a synthesizer and an I/Q demoduator was used to measure the complex transmission coefficient. Use of this configuration rather than a vector network analyzer allowed the excitation frequency to be swept in both the upward and downward directions by supplying a low-frequency $(<1 \mathrm{~Hz})$ triangle-wave voltage to the frequency modulation input of the synthesizer. It has been found in studies[1] of similar nonlinear su- 

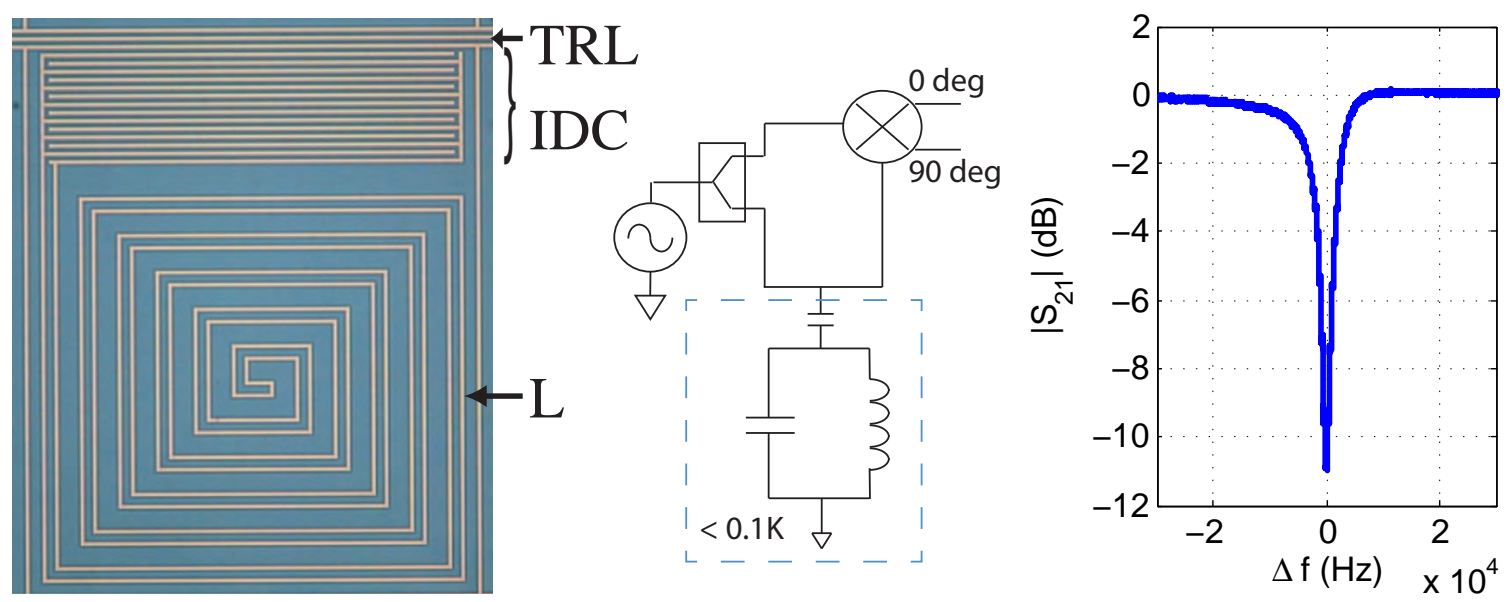

Figure S1: Microresonator geometry and circuit. (left) The lumped element resonator and microwave feedline (TRL) were patterned from single TiN and NbTiN films on high resistivity silicon substrates. The capacitor (IDC) of the resonator consists of planar interdigital electrodes, and the inductor (L) is a $5 \mu \mathrm{m}$ wide, $50 \mathrm{~nm}$ thick trace arranged in a (magnetically) noninductive spiral. The transition temperatures of the TiN and NbTiN films were $4 \mathrm{~K}$ and $14 \mathrm{~K}$ respectively. (center) A homodyne detector circuit measures the complex transmission of the resonator. The equivalent circuit of the resonator is a capacitively coupled tank circuit that shunts the readout transmission line, producing a dip in the transmission at the resonance frequency of $2.2 \mathrm{GHz}$ (right).

perconducting resonators that at large enough drive power the system undergoes a bifurcation beyond which the resonance curves exhibit discontinuous jumps and become hysteretic. This behavior occurs whenever the nonlinear dissipation is sufficiently low[1]. Beyond the bifurcation power, the resonance is more thoroughly explored by downward frequency sweeps, as these track the resonance frequency as it is shifted downward by the nonlinearity. For this reason, we concentrate here on the downward sweeping direction.

The data in fig. S2 show the highly distorted resonance curves that result when the resonator is driven well beyond the bifurcation. The behavior can be modeled using a current dependent shift of the resonance frequency $\delta f_{r}(I)$ and internal quality factor $Q_{i}(I)$ [1], where $I$ is the microwave current circulating in the resonator. In terms of these functions, the feedline transmission is

$$
S_{21}(f, I)=1-\frac{Q_{t}(I) / Q_{c}}{1+2 i Q_{t}(I)\left[f_{0}+\delta f_{r}(I)-f\right] / f_{0}},
$$

where $Q_{r}^{-1}(I)=Q_{i}^{-1}(I)+Q_{c}^{-1}$. The internal current can be expressed in terms of the feedline 


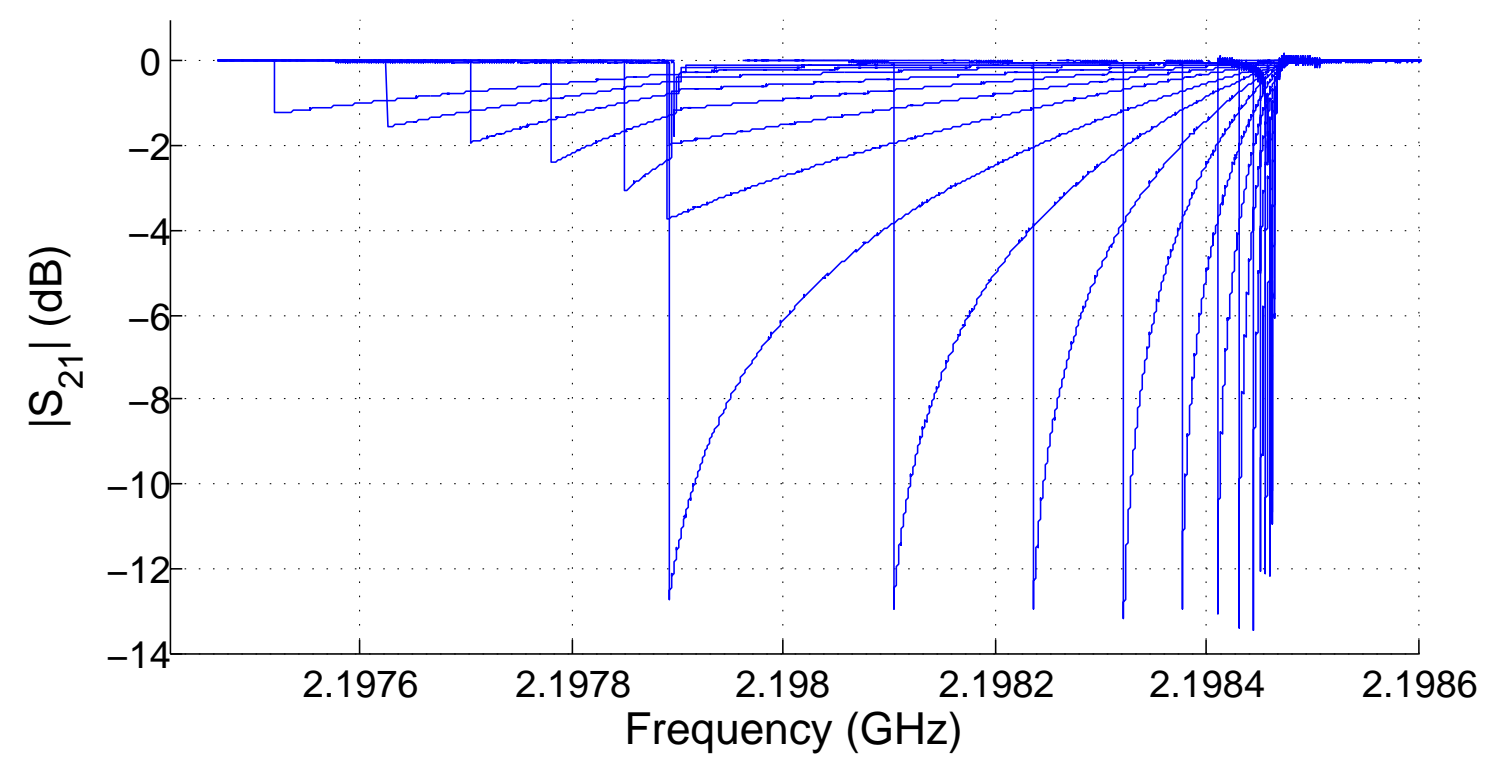

Figure S2: This figure illustrates the effect of increasing microwave readout power on the resonance line shape of the $2.2 \mathrm{GHz}$ lumped-element TiN resonator. As the power is increased, the microwave current in the resonator causes the inductance to increase, shifting the frequency downwards; this is classic "soft-spring" Duffing oscillator behavior. Interestingly, as indicated by the constant depth of the resonance, dissipation of the resonator changes very little over a wide range of drive power - in fact, the change in resistance of the TiN is $<2 \times 10^{-4}$ smaller than the change in its reactance in this range. Feedline powers are -46 to $-84 \mathrm{dBm}$ in steps of $-2 \mathrm{dBm}$. The current in the resonator is $\sim 1 \mathrm{~mA}$ at the onset of dissipation.

drive power $P_{f}$ and $S_{21}$ as

$$
I^{2}=2 Q_{c} \frac{\left|1-S_{21}\right|^{2}}{Z_{0}} P_{f},
$$

where $Z_{0}$ is the impedance of the resonator. The implicit set of equations $\mathrm{S} 1$ and $\mathrm{S} 2$ can be solved for $S_{21}$. For a downward frequency sweep, at a particular feedline power, the minimum of $\left|S_{21}\right|$ corresponds to the shifted resonance frequency $f_{r}(I)=f_{0}+\delta f_{r}(I)$. At these points $S_{21}$ is real and is equal to $1-Q_{r}(I) / Q_{c}$. For upward sweeps past the bifurcation power, $S_{21}$ never becomes real, as the system jumps discontinuously over the resonance condition.

Over a range of drive power the resonance dips shown in fig. S2 have approximately constant depth, hence the resonator dissipation remains unchanged in this range. In fact, no additional dissipation is observed up to a drive power which is approximately 100 times the bifurcation power. On further increasing the feedline power, the resonance dip does abruptly become much shallower, indicating a sudden onset of dissipation. Estimation of 


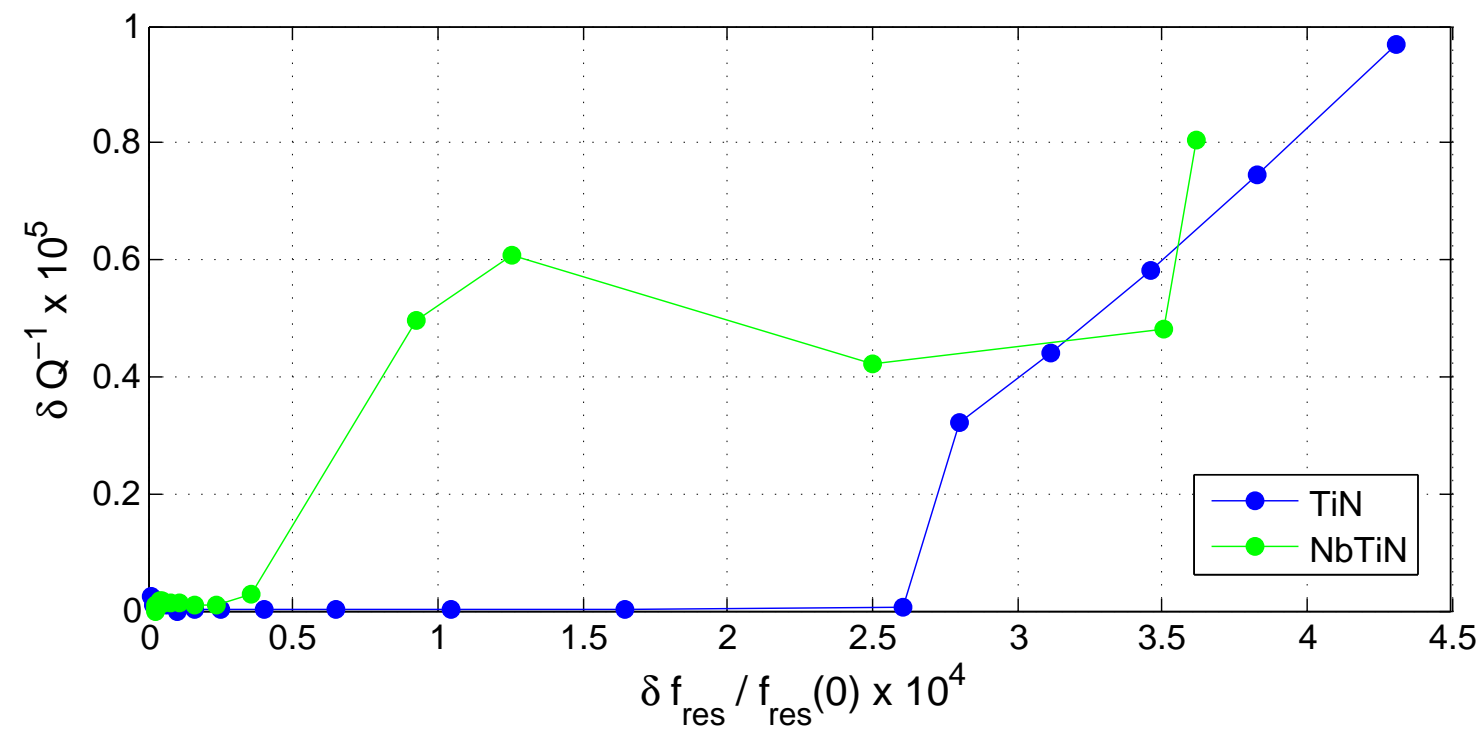

Figure S3: Frequency shift versus change in loss of TiN and NbTiN resonators at the shifted resonance frequencies for various values of the feedline power. For $\operatorname{TiN}, Q^{-1}$ remains unchanged to the measurement precision until $\delta f_{\text {res }} / f_{\text {res }}(0)=2.6 \times 10^{-4}$, which corresponds to a internal circulating current of $\approx 1 \mathrm{~mA}$. Beyond that point the loss increases roughly linearly with the frequency shift with a slope $\delta Q^{-1} /(\delta f / f)$ consistent with an increase in the quasiparticle density in the film. The $\mathrm{NbTiN}$ resonator shows a reduced range with no dissipation increase and a more complicated behavior at larger nonlinearity. For both resonators the loss remains less than $10^{-5}$ over the range studied.

the resonator microwave current at that point gives $I \approx 1 \mathrm{~mA}$.

Figure fig. S3 shows the change in the resonator loss $\delta Q^{-1}$ versus resonance frequency shift for the data of fig. S2 and for similar measurements on an NbTiN resonator. For both materials there is a range of drive power over which there is no increase the dissipation, to within the measurement accuracy, while significant resonance frequency shifts are observed. This behavior is in stark contrast to the $\mathrm{NbN}$ films used by Abdo et al. [2] and other studies [3-5] which show strong increases in dissipation as the power is increased.

The magnitude of the nonlinearity reached before dissipation is observed is $\xi=\delta f_{\text {res }} / f_{\text {res }}=$ $2.6 \times 10^{-4}$ for the TiN resonator. If we wish to operate the parametric amplifier below this limit, the value of $\xi$ would translate into a requirement on the transmission line length. Expressing the gain using the approximation $f_{s} \approx f_{p}$ and assuming perfect phase match $\Delta \beta=-2 \Delta \theta / L$, we expect $G_{s}=\exp (2 \xi \theta) / 4$, where $\theta=2 \pi L / \lambda_{g}$ is the phase length in ra- 
dians of the transmission line with physical length $L$ and guide wavelength $\lambda_{g}[6]$. For $20 \mathrm{~dB}$ gain, we would need $\theta \approx 3 \xi^{-1}$, a readily realizable length for frequencies in the $\mathrm{GHz}$ range, especially given that the phase velocity on the transmission line may be $\leq 0.1 c$. Note that the amplifier may also be operated at higher nonlinearity, as long as the increase in nonlinear dissipation remains small, as it does over the entire range of the data in the figure. In addition to the loss of the superconductor, the tones propagating on the transmission line will be attenuated by losses including dielectric loss, which is dominated by two-level systems[7] at very low temperature. This loss will be completely negligible because $\xi Q_{i} \gg 1$, as can be seen by noting that $Q_{i}>10^{6}$ for resonators made from TiN[8] or NbTiN[9].

\section{Coupled mode equations for predicting the gain of the paramp}

The wave equation for the current $I$ in the transmission line is

$$
\frac{\partial^{2} I}{\partial z^{2}}-\frac{\partial}{\partial t}\left[\mathcal{L}(I) \mathcal{C} \frac{\partial I}{\partial t}\right]=0
$$

where $\mathcal{L}(I)$ and $\mathcal{C}$ are the inductance and capacitance per unit length, and the current dependence of the inductance is given by

$$
\mathcal{L}(I)=\mathcal{L}_{0}\left(1+\frac{I^{2}}{I_{*}^{2}}\right)
$$

As in the standard treatment of waves interacting in nonlinear optical media, we express the total current in terms of a number of frequency components,

$$
I=\frac{1}{2}\left(\sum_{n} A_{n}(z) e^{i\left(k_{n} z-\omega_{n} t\right)}+\text { c.c. }\right),
$$

where the slowly varying complex mode amplitudes $A_{n}$ satisfy

$$
\left|\frac{d^{2} A_{n}}{d z^{2}}\right| \ll\left|k_{n} \frac{d A_{n}}{d z}\right| .
$$

The $I^{2} d I / d t$ nonlinearity connects combinations of four frequencies. Hence a general discussion of parametric amplification in a Kerr medium includes four frequencies in the sum in eqn. S5: two pump tones at $\omega_{p 1}$ and $\omega_{p 2}$, a weak signal at $\omega_{s}$ and a generated idler at 
$\omega_{i}=\omega_{p 1}+\omega_{p 2}-\omega_{s}$. Here we specialize to the case of degenerate four-wave mixing, $\omega_{p 1}=\omega_{p 2}$. Evolution of the mode amplitudes $A_{p}, A_{s}$ and $A_{i}$ inside the transmission line is then governed by three coupled mode equations[10],

$$
\begin{aligned}
\frac{d A_{p}}{d z} & =\frac{i k_{p}}{I_{*}^{\prime 2}}\left[\left(\left|A_{p}\right|^{2}+2\left|A_{s}\right|^{2}+2\left|A_{i}\right|^{2}\right) A_{p}+2 A_{s} A_{i} A_{p}^{*} e^{i \Delta \beta z}\right], \\
\frac{d A_{s}}{d z} & =\frac{i k_{s}}{I_{*}^{\prime 2}}\left[\left(\left|A_{s}\right|^{2}+2\left|A_{i}\right|^{2}+2\left|A_{p}\right|^{2}\right) A_{s}+A_{i}^{*} A_{p}^{2} e^{-i \Delta \beta z}\right], \\
\frac{d A_{i}}{d z} & =\frac{i k_{i}}{I_{*}^{\prime 2}}\left[\left(\left|A_{i}\right|^{2}+2\left|A_{s}\right|^{2}+2\left|A_{p}\right|^{2}\right) A_{i}+A_{s}^{*} A_{p}^{2} e^{-i \Delta \beta z}\right],
\end{aligned}
$$

where the low-power propagation mismatch $\Delta \beta=k_{s}+k_{i}-2 k_{p}$, and $I_{*}^{\prime 2}=2 I_{*}^{2} / \alpha$.

The number of equations in S7 could be increased if we were to include mixing processes involving, for example, the pump harmonics $(2 n+1) \omega_{p}, n=1,2 \ldots$, and combinations such as $2 n \omega_{p}+\omega_{s}$, etc. We assume here that these higher frequency tones either fall in a stop band of the stepped impedance structure and do not propagate, or at least that the dispersion engineering of the line creates enough phase mismatch at those frequencies that they do not interact coherently with the lower frequency modes.

For $\omega_{s} \approx \omega_{p}$ and using the undepleted pump approximation, $d\left|A_{p}\right| / d z=0$, eqns. S7 yield analytical results for the signal power gain $G_{s}$ and idler conversion efficiency $G_{i}$, which may be expressed as $[6,11]$

$$
\begin{aligned}
G_{s} & =\frac{\left|A_{s}(L)\right|^{2}}{\left|A_{s}(0)\right|^{2}}=1+\left[\frac{k_{p}\left|A_{p}\right|^{2}}{I_{*}^{\prime 2} g} \sinh g L\right]^{2} \\
G_{i} & =G_{s}-1
\end{aligned}
$$

with parametric gain coefficient $g=-\Delta \beta\left(\Delta \beta / 4+k_{p}\left|A_{p}\right|^{2} / I_{*}^{\prime 2}\right)$. In the case of no dispersion, $\Delta \beta=0, g=0$ implies that

$$
G_{s}=1+\left(\frac{k_{p}\left|A_{p}\right|^{2}}{I_{*}^{\prime 2}}\right)=1+(\Delta \theta)^{2},
$$

where $\Delta \theta$ is the phase shift of the pump tone. The gain in this case is quadratic in the length of the transmission line, rather than exponential, owing to a phase mismatch that results from the nonlinearity (as can be seen from the factors of 2 in eqn. S7, the phase shifts of the 

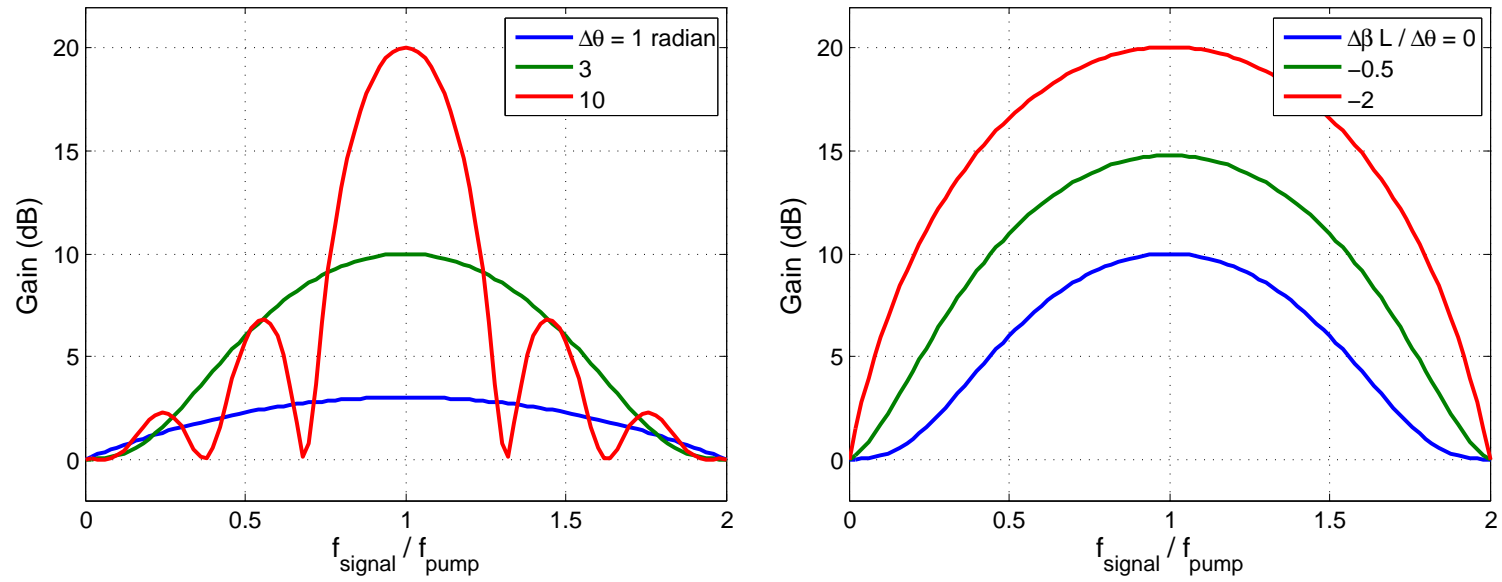

Figure S4: Calculation of the paramp gain using the coupled mode equations S7 for (left:) no dispersion and various values of the nonlinear phase shift $\Delta \theta$; and (right:) fixed $\Delta \theta=$ 3 radians and various values of $\Delta \beta$.

signal and idler tones are twice that of the pump tone.) If $\Delta \beta=-k_{p}\left|A_{p}\right|^{2} / I_{*}^{\prime 2}$, the linear phase mismatch compensates that due to the nonlinearity and the exponential gain regime is accessed with

$$
G_{s} \approx \frac{1}{4} \exp (2 \Delta \theta)
$$

The kinetic inductance paramp described in this paper may operate well outside the frequency range where $\omega_{s} \approx \omega_{p}$ is valid, so to predict the gain and bandwidth we integrate eqns. S7 numerically. The results of that calculation for several idealized cases are shown in fig. S4. Here we assume that the frequencies other than those of the pump, signal and idler are blocked by engineered stop bands or large dispersion at those frequencies. For simplicity, we ignore the narrow stop band near the pump that would be associated with introducing dispersion at that frequency. We also neglect the detailed form of the dispersion near $\omega_{p}$, taking $\Delta \beta$ to be either a negative constant or zero. The calculation results uphold the very broadband nature of the amplifier. In the case of no dispersion, the 3 - $\mathrm{dB}$ bandwidth narrows, but remains above $25 \%$ for $20 \mathrm{~dB}$ peak gain. For optimal $\Delta \beta, 20 \mathrm{~dB}$ peak gain may be realized with a phase shift of only 3 radians, and the $3-\mathrm{dB}$ bandwidth is greater than an octave. 


\section{Device fabrication}

The NbTiN itself is deposited by dc reactive magnetron- sputtering from a 3 inch diameter NbTi metal target. The films are sputtered using constant current (.55-.8A) in a background of Argon and Nitrogen. The process pressure is maintained at 5 mTorr using a downstream conductance controller and the flow rates are set using mass flow controllers. The deposition system is a load-locked UHV system with a base pressure $\sim 5 \times 10^{-10}$ Torr. The TiN films are deposited following the procedures discussed in [8].

\section{References}

1. Yurke, B. \& Buks, E. Performance of cavity-parametric amplifiers, employing Kerr nonlinearites, in the presence of two-photon loss. J. Lightwave Technol. 24, 5054-5066 (Dec. 2006).

2. Abdo, B., Segev, E., Shtempluck, O. \& Buks, E. Nonlinear dynamics in the resonance line shape of NbN superconducting resonators. Phys. Rev. B 73, 134513 (2006).

3. Dahm, T. \& Scalapino, D. J. Theory of intermodulation in a superconducting microstrip resonator. J. App. Phys. 81, 2002-2009 (Feb. 1997).

4. Golosovsky, M. A., Snortland, H. J. \& Beasley, M. R. Nonlinear microwave properties of superconducting Nb microstrip resonators. Phys. Rev. B 51, 6462-6469 (Mar. 1995).

5. Chin, C. C., Oates, D. E., Dresselhaus, G. \& Dresselhaus, M. S. Nonlinear electrodynamics of superconducting $\mathrm{NbN}$ and $\mathrm{Nb}$ thin films at microwave frequencies. Phys. Rev. B 45, 4788-4798 (Mar. 1992).

6. Hansryd, J, Andrekson, P., Westlund, M, Li, J \& Hedekvist, P. Fiber-based optical parametric amplifiers and their applications. IEEE J. Sel. Top. Quantum Electron. 8, 506-520. ISSN: 1077-260X (2002).

7. Gao, J. et al. Experimental evidence for a surface distribution of two-level systems in superconducting lithographed microwave resonators. Appl. Phys. Lett. 92, 152505 (Apr. 2008).

8. Leduc, H. G. et al. Titanium nitride films for ultrasensitive microresonator detectors. App. Phys. Lett. 97, 102509 (Sept. 2010).

9. Barends, R. et al. Contribution of dielectrics to frequency and noise of NbTiN superconducting resonators. Appl. Phys. Lett. 92, 223502 (2008).

10. Agrawal, G. P. Applications of nonlinear fiber optics (ed Agrawal, G. P.) (2001).

11. Stolen, R. \& Bjorkholm, J. Parametric amplification and frequency conversion in optical fibers. IEEE J. Quantum Electron. 18, 1062 -1072. ISSN: 0018-9197 (1982). 